\title{
Nanostructured Materials for Clean Energy and Environmental Challenges
}

\author{
Hui Xia, ${ }^{1}$ Michael Z. Hu, ${ }^{2}$ Ying Shirley Meng, ${ }^{3}$ Jianping Xie, ${ }^{4}$ and Xiangyu Zhao ${ }^{5}$ \\ ${ }^{1}$ School of Materials Science and Engineering, Nanjing University of Science and Technology, Nanjing 210094, China \\ ${ }^{2}$ Oak Ridge National Laboratory, Oak Ridge, TN 37831-6181, USA \\ ${ }^{3}$ Department of NanoEngineering, University of California, San Diego, CA 92093-0448, USA \\ ${ }^{4}$ Department of Chemical and Biomolecular Engineering, National University of Singapore, Singapore 117576 \\ ${ }^{5}$ Institute of Nanotechnology, Karlsruhe, Institute of Technology, Karlsruhe, Germany
}

Correspondence should be addressed to Hui Xia; xiahui@njust.edu.cn

Received 18 February 2014; Accepted 18 February 2014; Published 23 March 2014

Copyright (C) 2014 Hui Xia et al. This is an open access article distributed under the Creative Commons Attribution License, which permits unrestricted use, distribution, and reproduction in any medium, provided the original work is properly cited.

Increasing supply of energy is required to cater the rapid economic growth, which has posed a huge burden on the current energy infrastructure and the environment. The excessive consumption of limited fossil fuel tends to dry up the energy sources and causes severe environmental pollution and global warming. Such challenging issues have motivated the scientific community to seek next generation energy sources and to develop innovative methodologies to address the key environmental issues to realize a sustainable future. Nanostructured materials possess intriguing physical and chemical properties and have recently emerged as ideal platforms to solve many key challenges in energy (e.g., energy conversion (solar cells and fuel cells) and energy storage (lithium-ion batteries and supercapacitors)) and environment (e.g., green catalysts, sensors, pollution prevention, treatment, and remediation). In particular, nanostructured materials feature with controllable size, shape, composition, structure, and surface, which are difficult or impossible to achieve in their bulk materials. Such unique features of nanostructured materials can be further tailored and engineered to specifically tackle a particular energy and/or environmental challenge.

In this special issue, 14 original research papers are presented, bringing over recent advances and $\mathrm{R} \& \mathrm{D}$ activities in developing nanostructured materials for groundbreaking applications in energy and environment. In the paper entitled "CuO-In $\mathrm{O}_{3}$ core-shell nanowire based chemical gas sensors," $\mathrm{X}$. $\mathrm{Li}$ et al. reported the synthesis of $\mathrm{CuO}-\mathrm{In}_{2} \mathrm{O}_{3}$ core-shell nanowires, which exhibit improved sensing performance to hydrogen and propane as a result of the coated porous $\mathrm{In}_{2} \mathrm{O}_{3}$ shell and the p-n junction formed at the core-shell interface. A plasmonic effect of $\mathrm{Ag}$ nanoparticles in the dye-sensitized solar cells has been confirmed in the paper "Solution processed silver nanoparticles in dye-sensitized solar cells" by Berginc et al. In the paper entitled "High photocatalytic activity of $\mathrm{Fe}_{3} \mathrm{O}_{4}-\mathrm{SiO}_{2}-\mathrm{TiO}_{2}$ functional particles with core-shell structure," $\mathrm{C}$. Xue et al. reported a novel method of synthesizing $\mathrm{Fe}_{3} \mathrm{O}_{4}-\mathrm{SiO}_{2}-\mathrm{TiO}_{2}$ functional particles with high photocatalytic activity under visible light. In the paper entitled "Structural evolution and electrochemical performance of $\mathrm{Li}_{2} \mathrm{MnSiO}_{4} / \mathrm{C}$ nanocomposite as cathode material for Li-ion batteries," M. Wang et al. reported that the solgel method synthesized $\mathrm{Li}_{2} \mathrm{MnSiO}_{4} / \mathrm{C}$ nanocomposite with a large reversible capacity of $257 \mathrm{mAhg}^{-1}$ and good rate capability. P. Yu et al. reported Y-doped $\mathrm{ZnO}$ nanorods by hydrothermal method and their acetone gas sensitivity. In the rest of papers in this special issue, M. Israelowitz et al. reported spin coated plasmonic nanoparticle interfaces for photocurrent enhancement in thin film Si solar cells, F. Wang et al. reported the preparation and performance of inorganic heat insulation panel based on sepiolite nanofibers, and they also reported the preparation and performance of cis-polybutadiene rubber composite materials reinforced by organic modified palygorskite nanomaterials. J. Xu et al. reported the field emission properties of the graphene-double walled carbon nanotube hybrid films prepared by vacuum 
filtration and screen printing, Z. Dai et al. reported the development of amperometric laccase biosensor through immobilizing enzyme in magnesium-containing mesoporous silica sieve (Mg-MCM-41)/polyvinyl alcohol (PVA) matrix, $\mathrm{L}$. Li et al. reported pores and microanalysis of microbeinspired nano- $\mathrm{CaCO}_{3}$ cementing sand columns, P. Zhang et al. reported a study on notch sensitivity of fracture properties of concrete containing nano- $\mathrm{SiO}_{2}$ particles and fly ash, $\mathrm{X}$. Shi et al. reported mesoporous alumina microfibers in situ transformation from AACH fibers and the adsorption performance, and $\mathrm{T}$. Wu et al. reported electroreduction of copper dichloride powder to copper nanoparticles in an ionic liquid.

We are pleased to see the progress on design, synthesis, characterization, and applications of nanostructured materials in energy and environment. We hope that this special issue will promote further investigation on advanced nanostructured materials to develop the next generation energy sources and innovative solutions to realize a sustainable future.

\section{Acknowledgments}

The editors would like to express their sincere appreciation to all authors for their contributions and to all reviewers for their time and constructive comments for this special issue.

Hui Xia

Michael Z. Hu

Ying Shirley Meng

Jianping Xie

Xiangyu Zhao 

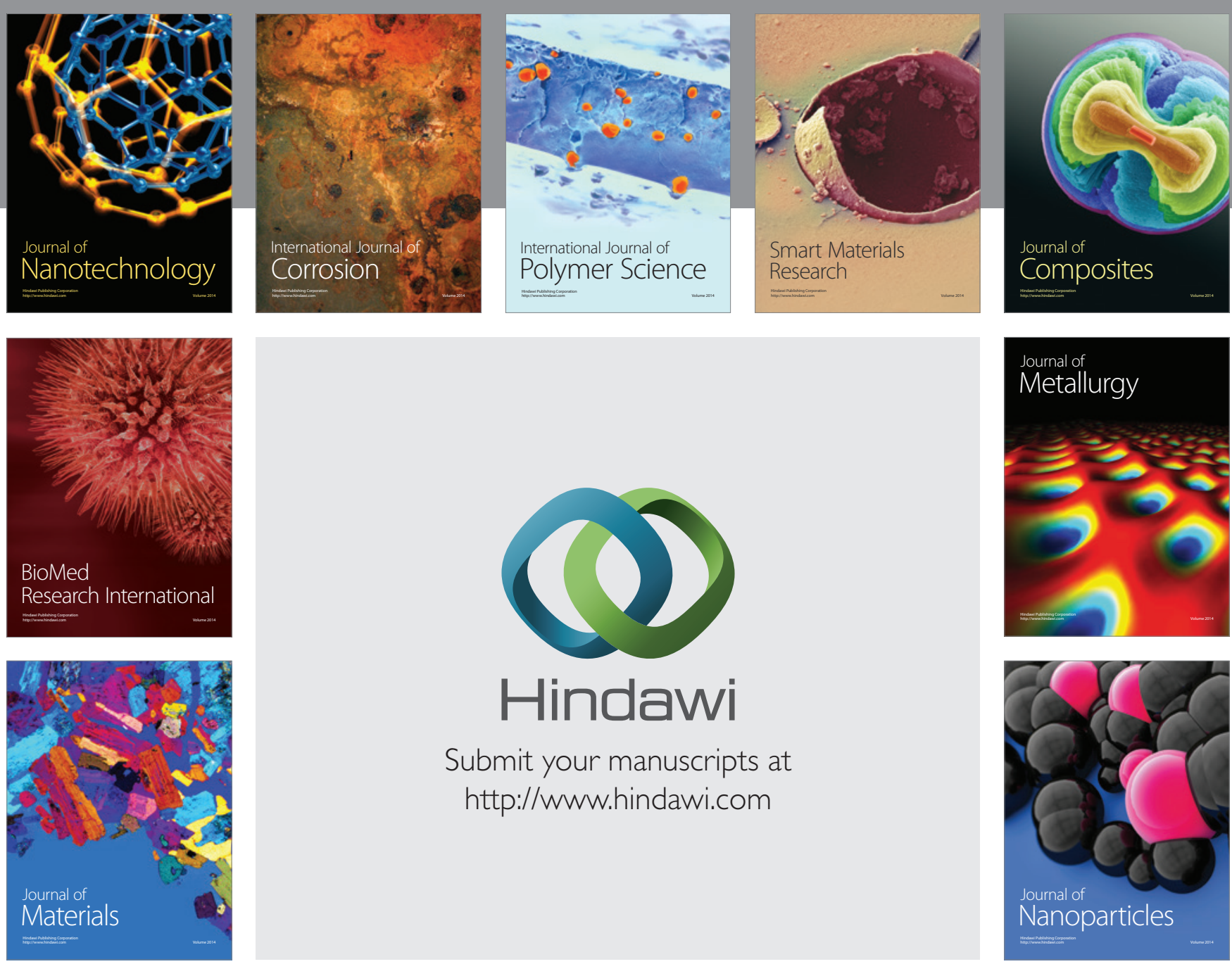

Submit your manuscripts at http://www.hindawi.com
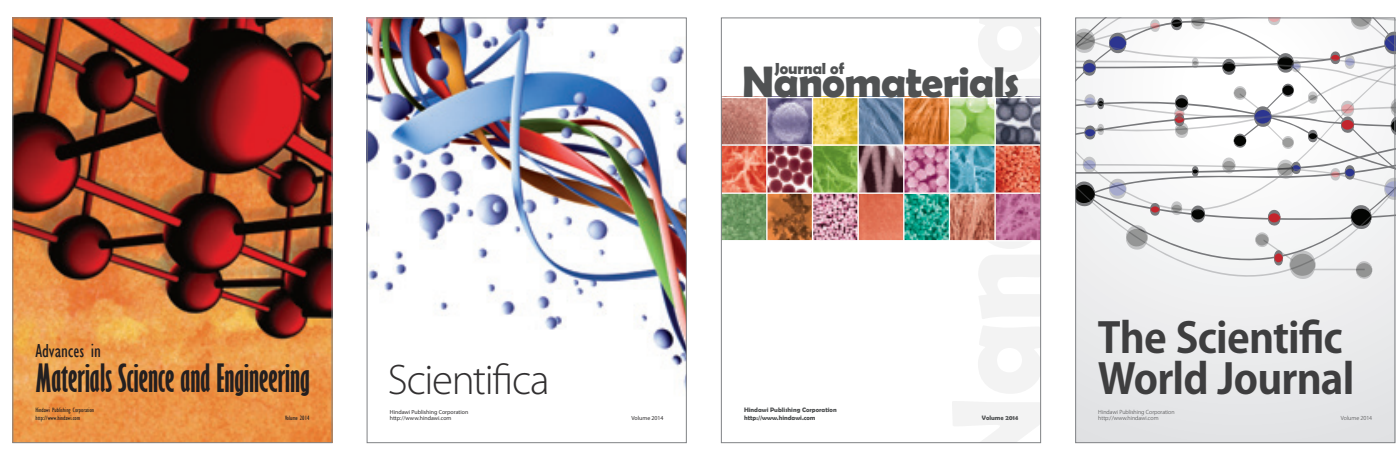

\section{The Scientific World Journal}
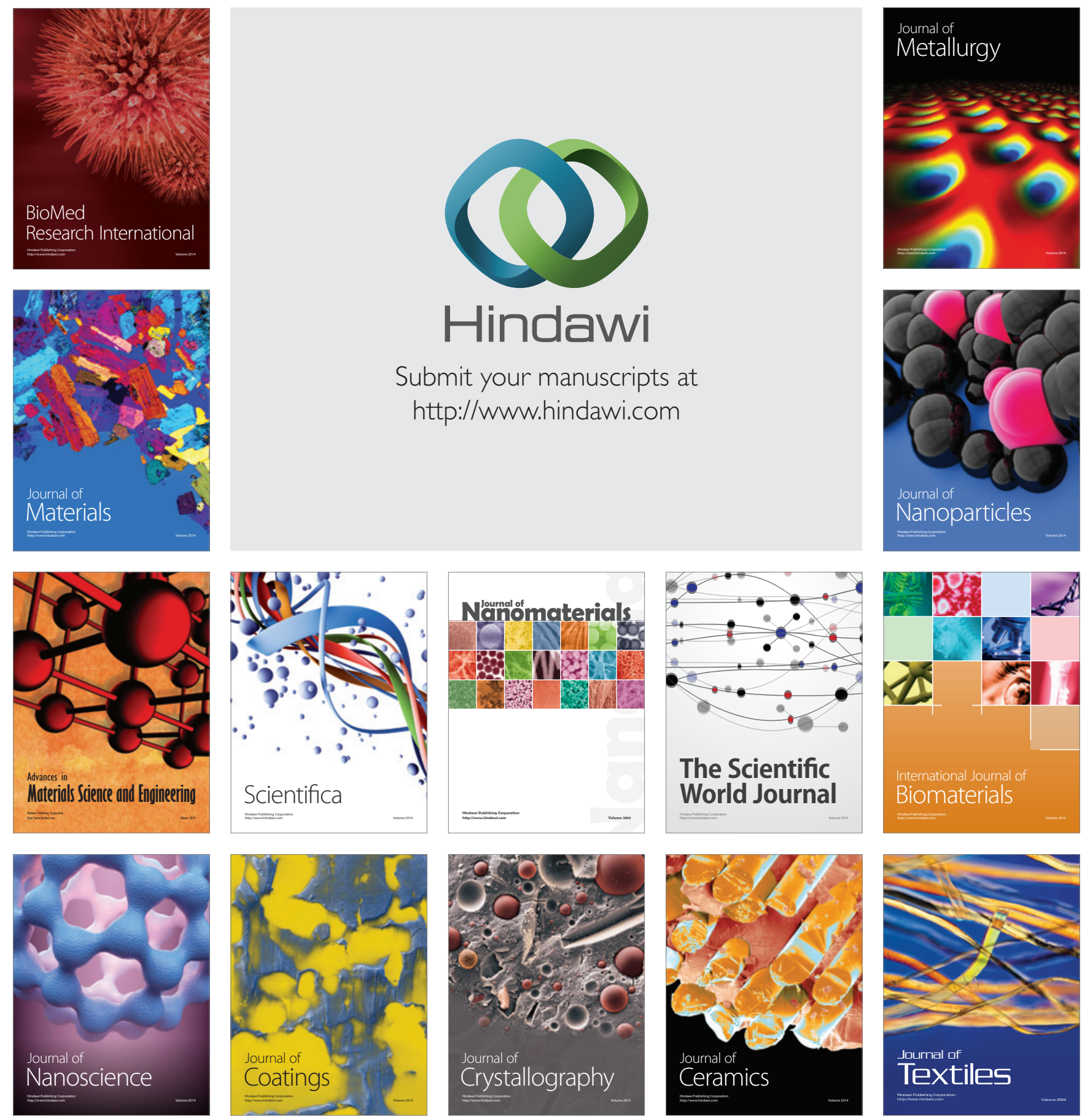